\title{
APPROACHES TO AUTOMATICALLY EXTRACT AFFORDANCES FROM PATENTS
}

\author{
Chiarello, Filippo (1); Cirri, Ilenia (1); Melluso, Nicola (1); Fantoni, Gualtiero (1); Bonaccorsi, \\ Andrea (1); Pavanello, Tommaso (2) \\ 1: Università di Pisa; 2: Erre Quadro s.r.1.
}

\begin{abstract}
The importance of affordance in Engineering design is well established. Artifacts that are able to activate spontaneous and immediate users' reactions are considered the outcome of good design practice.

A huge effort has been made by researchers for understanding affordances: yet these efforts have been somewhat elusive. In particular, they have been limited to case studies and experimental studies, usually involving a small subset of affordances. No systematic effort has been carried out to list all known affordance effects. This paper offers preliminary steps for such an ambitious effort.

We propose a set of three different approaches of Natural Language Processing techniques to be used to extract meaningful affordance information from the full text of patents: 1) a simple word search, 2) a lexicon of affordances and 3) a rule-based system.

The results give in-depth measures of how rare affordances in patents are, and a fine grain analysis of the linguistical construction of affordances. Finally, we show an interesting output of our method, that has detected affordances for disabled people, showing the ability of our system to automatically collect design-relevant knowledge.
\end{abstract}

Keywords: Affordance, Patents Analysis, Design theory, Semantic data processing, Design methods

\section{Contact:}

Chiarello, Filippo

Università di Pisa

Italy

filippochiarello.90@gmail.com

Cite this article: Chiarello, F., Cirri, I., Melluso, N., Fantoni, G., Bonaccorsi, A., Pavanello, T. (2019) ‘Approaches to Automatically Extract Affordances from Patents', in Proceedings of the 22nd International Conference on Engineering Design (ICED19), Delft, The Netherlands, 5-8 August 2019. DOI:10.1017/dsi.2019.255 


\section{INTRODUCTION}

The importance of affordance in Engineering design is well established. Artifacts that are able to activate spontaneous and immediate users' reactions, leading to a smooth and intuitive use of the object are considered the outcome of good design practice.

For this reason many studies in Cognitive Psychology have addressed the causes and consequences of affordance and many others in Engineering Design have explored the possibility to improve the design practice in order to capture the great benefits of user experience behind affordances.

Yet these efforts have been somewhat elusive. In particular, they have been limited to case studies and experimental studies, usually involving a small subset of affordances. No systematic effort has been carried out to create a large scale repository, or knowledge base, for all known affordance effects.

This paper offers the preliminary steps for such an ambitious effort.

It advocates the use of patent texts as the main source of information, and of Natural Language Processing (NLP) techniques to be used to extract meaningful affordance information from the full text.

After a short review of the literature, the paper critically discusses the state of the art in information retrieval of affordances (Section 2), showing its serious limitations. It then explores concurrent NLP approaches (Section 3), discussing their theoretical background, preliminary results and limitations. Section 4 shows an interesting preliminary finding about affordances for people with disabilities. Finally section 5 discusses the main conclusion of the work and proposes some further research path.

\section{LITERATURE REVIEW}

In the present section we present the literature that is relevant to our work. In section 2.1 we show the works that defined the concept of affordance; in section 2.2 we review the literature related to the extraction of design concepts from patents.

\subsection{Affordance: a concept between Engineering design and Cognitive psychology}

The notion of affordance has been extensively studied in Engineering design and Cognitive psychology, and more recently in Robotics and Artificial intelligence. According to the pioneering studies of Gibson (1966), "when the constant properties of constant objects are perceived (the shape, size, color, texture, composition, motion, animation, and position relative to other objects), the observer can go on to detect their affordances".

In addition, the cognitive processes that lead to affordances invite the actor to an action of use of the object (Gibson 2014). The subsequent literature has elaborated on this notion and in some case has significantly extended it.

Several authors (Cascini 2011, Gibson et al., 1994, Turvey 1992, Stoffregen 2003) agree with the common point that an affordance manifests itself in relation to the action and direct perception capabilities of a particular actor. This means that affordance is a relational concept, requiring the interaction between different entities, rather than a properties of entities themselves (object or actor).

Two mail lines of extension of the concept can be mentioned. First, while in the original formulation there was an emphasis on visual perception, some authors (Jamone et al., 2018) wonder why this should not extended to other sensory perceptions. For example, a computer fan provides auditory affordance to its user via its noise.

Second, following an early suggestion of Maier and Fadel (2003), Chemero (2003) has proposed an ecological perspective on affordance. He claims that affordances refer to the relation between the actor and the overall environment that interact with an object, not only with the individual object. In this contextual perspective, the proposition that describes the affordance relation between two entities of the type "The apple affords to be eaten by the pig" has the same structure than the proposition "John is taller than Mary".

Another group of researchers gives an interesting point of view on affordances (Maier and Fadel, 2003, Brown and Blessing, 2005, Gero and Kannengiesser, 2004, Kannengiesser and Gero, 2012). Following these contributions in Engineering Design theory, affordances can be seen as "cognitive shortcuts", or fast "cognitive processes that reduce the burden of reasoning". By these shortcuts the 
user skips the entire reasoning about the causality chain and links directly the behavior with the structural feature of the object (Spreafico et al., 2015).

The concept of "cognitive shortcut" shifts the affordances focus to the perspective of the user, who needs to perceive the features in the most disambiguous way. When a user is exposed to a certain number of the same events, manipulating homogeneous classes of objects, he or she immediately associates the structure of the object (or part of it) with its possible behaviours. However the affordance problem is designing common products in a way that they are accessible and easily usable by as many people as possible. The inclusiveness is an important challenge for everyday objects designing, but nowadays it is limited to specific cases of study and groups of users (such as Dong and Vanns 2009, Langdon et al., 2015 and Goodman-Deane et al., 2016).

While there is significant theoretical development on the concept of affordance, with significant agreement between studies in Cognitive psychology and in Engineering design, there is still a lot to be done to make the concept global and operational. In particular, what is needed is an effort to build up a repository, or a structured list of affordances, to be used for information search and retrieval, on the one hand, and for methods of creativity and conceptual design, on the other hand. In turn, this would require to identify the sources of information for such a large scale effort.

\subsection{Extracting design relevant information from patents texts}

While there is significant agreement on the concept of affordance and on its importance, there is little work on how to make it usable for pragmatic purposes. The starting point should be to ask about the sources of information needed to identify affordances.

We suggest that a promising source is the full text of patents. Patents cover approximately $80 \%$ of all technical information available. They are by nature publicly available and an impressive literature has been developed in the last few years to extract information from the text of patents, aimed at a variety of audiences, from readers interested in Strategic management and Technology management (Holger 2003) to marketers and designers (Chiarello 2017). In this paper we focus on information aimed at supporting the design process, from the conceptual stage down to industrial development. In this process affordance-related information would be of great value.

At the same time, it must be recognized that there are still large difficulties in extracting information from patents, in particular in identifying and extracting design related information. The main reason is that this information (e.g. the user of the invention, the advantages of the design solution described in the patent or the drawbacks of the state of the art of a class of products) are hidden in patents documents (Chiarello 2018). With the word hidden we mean that design related information in patents are:

- Rare: only few patent applicants insert this information in the patent application, since it is not mandatory for legal reasons.

- Fuzzy: even if an applicant insert design related information, the linguistic form in which this information is written is complex and obscure, since applicants try to make it hard for other designers or inventors to find the patent or to design around it.

This means that in order to extract valid information, dedicated analytical techniques must be designed and implemented. It is well known how Genrich Saulovich Altshuller for the development of TRIZ identified several design rules by manually inspecting thousands patents, in a fully bottom-up approach (Savransky 200). Luckily enough, impressive advancements in computational linguistics in the last two decades made it possible to carry out analysis on the full content of large collections of patent texts. It is clear that text mining analysis of patents can be a game changing source of information for designers (Boyack et al., 2013).

\section{APPROACHES TO FIND AFFORDANCES IN PATENTS}

In this section we explore three different approaches to find affordances in patents. We start with the simple case of a word search in 3.1, then in section 3.2 we study the development of a lexicon of affordances and finally in section 3.3 we create a rule-bases sentence extractor able to extract sentences containing affordances. 


\subsection{A text-search approach}

A first naïve approach to find affordances in patents is to search for the word affordance (and its lexical variations). The results of this search would also give us a preliminary metric of how hard is find affordances in patents. To do that we have to compare the volume of this search with another one and see if there are differences between patent classes. For this reason, table 1 shows the distribution of the term affordance and user within the International Patent Classification (the first 15 results in decreasing order of occurrence for the word affordance).

It is interesting to observe that, within the first 15 sub-classes, the class G06 (Computing; Calculating; Counting) occurs four times and the section $\mathrm{G}$ (Physics) ten times.

Furthermore, from these data it is possible to make the following observations:

- the term affordance appears very rarely, less than one in thousand cases

- it is not equally distributed across patent classes

- the presence of the term is concentrated in just one IPC class, which largely contains technological devices.

In comparison, searching for user shows that this term is significantly more used than affordance (on average, from $10 \%$ to $40 \%$ of patents). Accordingly it is possible to conclude that affordance-related terms are rarely used in patents in the explicit form. This makes the search for affordance-related words more difficult- but perhaps more rewarding if successful.

Table 1. Subset of "affordance" and "user" distribution over the IPC sub-classes

\begin{tabular}{|l|l|l|l|l|l|}
\hline Class Name & IPC & $\begin{array}{l}\text { All } \\
\text { patents }\end{array}$ & $\begin{array}{l}\text { Patents } \\
\text { including } \\
\text { affordances }\end{array}$ & $\begin{array}{l}\text { \% patents } \\
\text { including } \\
\text { affordances }\end{array}$ & $\begin{array}{l}\text { \% patents } \\
\text { including } \\
\text { users }\end{array}$ \\
\hline $\begin{array}{l}\text { Electric digital data } \\
\text { processing }\end{array}$ & G06F & $4,850,637$ & 2,797 & 0.0005 & 0.3728 \\
\hline $\begin{array}{l}\text { Transmission of digital } \\
\text { Information }\end{array}$ & H04L & $2,495,628$ & 532 & 0.0002 & 0.3468 \\
\hline $\begin{array}{l}\text { Data processing systems } \\
\text { or methods }\end{array}$ & G06Q & $1,104,958$ & 472 & 0.0004 & 0.4343 \\
\hline Pictorial communication & H04N & $2,742,473$ & 387 & 0.0001 & 0.2182 \\
\hline Image data processing & G06T & 844,257 & 310 & 0.0003 & 0.2352 \\
\hline $\begin{array}{l}\text { Telephonic } \\
\text { communication }\end{array}$ & H04M & 990,667 & 202 & 0.0002 & 0.3257 \\
\hline $\begin{array}{l}\text { Arrangements or circuits } \\
\text { for control }\end{array}$ & G09G & 634,288 & 197 & 0.0003 & 0.1946 \\
\hline $\begin{array}{l}\text { Wireless communications } \\
\text { Networks }\end{array}$ & H04W & $1,404,091$ & 197 & 0.0001 & 0.4094 \\
\hline Recognition of data & G06K & 991,586 & 194 & 0.0001 & 0.2679 \\
\hline $\begin{array}{l}\text { Speech analysis or } \\
\text { synthesis }\end{array}$ & G10L & 260,878 & 137 & 0.0005 & 0.2925 \\
\hline $\begin{array}{l}\text { Measuring distances, } \\
\text { levels or Bearings }\end{array}$ & G01C & 430,077 & 90 & 0.0002 & 0.1837 \\
\hline Information storage based & G11B & $1,418,776$ & 79 & 0.0001 & 0.0981 \\
\hline $\begin{array}{l}\text { Card, board, or roulette } \\
\text { games }\end{array}$ & A63F & 550,182 & 72 & 0.0001 & 0.1221 \\
\hline $\begin{array}{l}\text { Computer systems based } \\
\text { on specific computational } \\
\text { models }\end{array}$ & G06N & 96,705 & 70 & 0.0007 & 0.3903 \\
\hline $\begin{array}{l}\text { Signalling or calling } \\
\text { systems }\end{array}$ & G08B & 455,249 & 68 & 0.0001 & 0.1937 \\
\hline
\end{tabular}


Analysing the problem more in detail, figure 1 shows that, while the absolute occurrence of userrelated and affordance-related words differs by several orders of magnitude, the two classes of words are correlated.

Patent sub-classes located in the green region refer to Transmission of digital communication and Data processing. They exhibit a larger occurrence of user-related words, perhaps as a consequence of the intense use of the word "user" in the technical language of Information technology. Another interesting part of the figure is the purple region. It shows that there are not patent sub-classes in which affordance-related terms are used without the user-related ones. In the blue region, it is reasonable to see the class Speech analysis or synthesis, where the uncanny valley (Mori, 1970) of bad designed affordances can deeply affect the usability of such systems. We can thus summarise that the behaviour of the term affordance is anomalous de facto since it is explicit in classes more related to communication and information than in classes related to standard artefacts. Such patents contain attempts to recreate "the affordance of an object in the virtual world", replicate a number of affordances (of paper) that cannot be easily replaced with existing digital media (G06K), improve the UI via an intuitive "user interface affordance" (G06T), with "a control affordances widget containing a set of control buttons" (G06N), "a GUI affordance (such as a scroll bar) with which the off-screen objects are to moved into view" (G09G).

Probably (see next paragraphs) the affordances in cases of artefacts are more explicit and the affordance is described more with his -ability than with the term affordance itself.

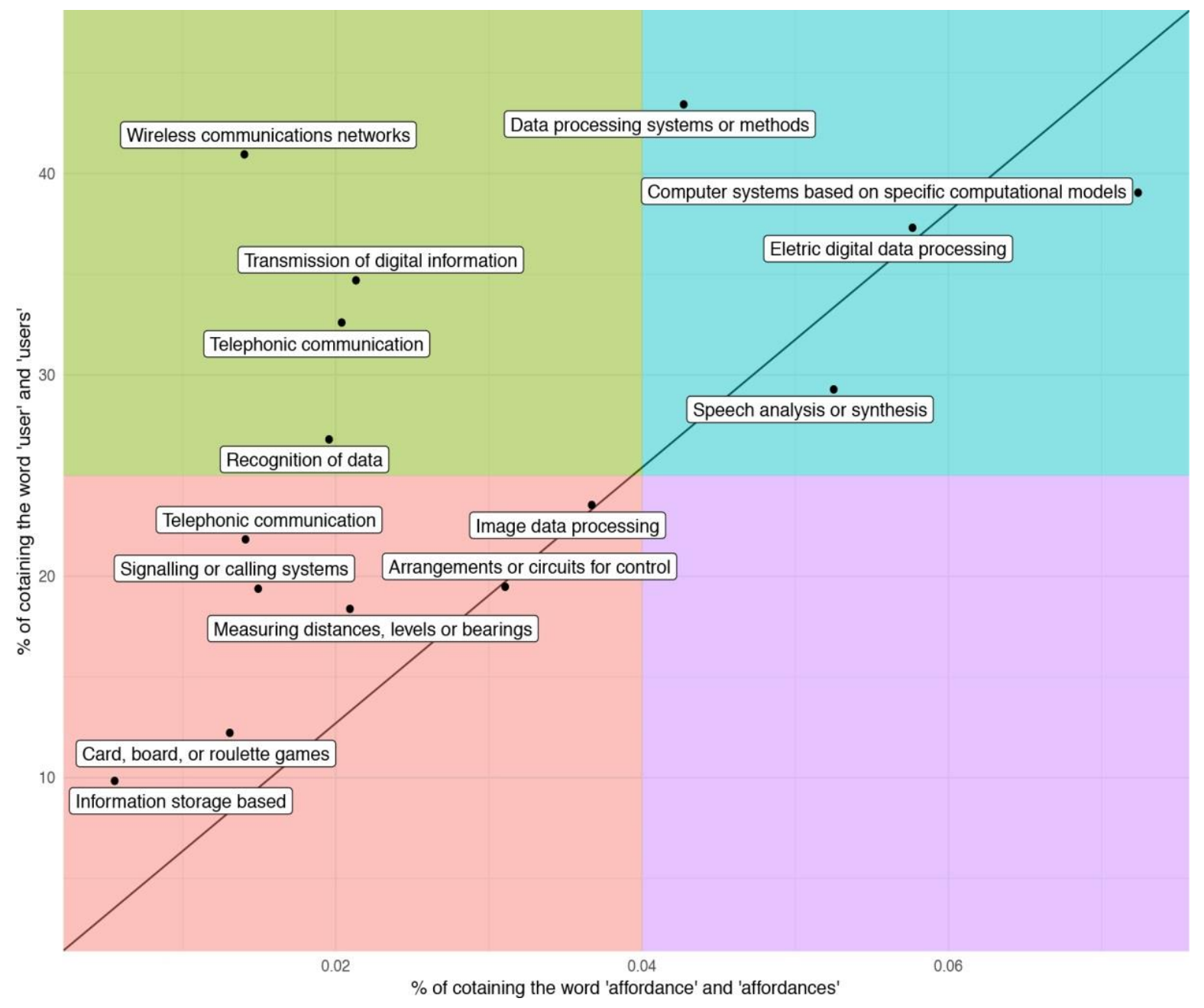

Figure 1. Correlation between the percentages of occurrence of user-related and affordance-related words across patent sub-classes 


\subsection{Developing a lexicon of affordances: the - Ility approach}

Several authors (Maier and Fadel, 2003, Kannengiesser and Gero, 2012, Spreafico et al., 2015, Jamone et al., 2018) uses the suffix -ability in order to indicate affordances. For example the stairs afford climbability while the chairs afford sittability. In the present section we study the implications of using this lexical construction to automatically extract affordances from patents.

First of all, we have to consider the fact that the collection of words ending with -ability can lead to extract false positives: collecting all the English words ending in - ability is not a suitable strategy. For example stability is in between a property and an affordance, but only the context helps to discriminate between the two. Probability, disability, viability and many others certainly do not indicate an affordance.

In order to eliminate this problem we carried out an extensive domain knowledge-based analysis of correctly expressed affordances ending with -ability suffix. This analysis leads to the following restriction: $a$ word ending with the suffix-ability is an affordance if and only if it includes a functional verb. For example storability is an affordance because to store is a functional verb.

The theoretical rationale for this suggestion comes from the idea that affordances were functions in the past, but have become affordances due to repeated use. The user immediately connects the implicit function to one or more product features, without reflecting on the abstract function implied by the use. In order to reconstruct the origins of the affordance we should start from a careful consideration of those verbs that are implicit in words ending with -ability and select only those with a functional content. As we will see, this process can be automatized to a large extent.

At the same time the collection of words ending with -ability can lead to false negative too. In fact it ignores those expression with functional verbs + ability than cannot be properly expressed in the English language. Many functional verbs simply do not have an English word that transform them into an -ability word. Anyway, the constructs ability to, able to, capability of, capable of, easy to plus functional verbs are in part used to solve some of these language problems. Therefore affordances are more than the -ability ending words and an extensive and detailed study about their presence in patents can not ignore such constructs.

Summing up, the candidate collection of words denoting affordances is formed by the subset of words ending in -ability in which the verb is functional, plus those expressions in which functional verbs appear after supporting expressions that denote a capability.

It should be noted that this collection may still include ambiguities. In some cases we can have affordances in the verb + ability structure even if the verb is not strictly functional verbs, at least in the rigorous definition of Stone and Wood (2000) and Pahl and Beitz (2013). For example, to climb or to sit are not functional verbs, but rather denote the intention of the user. Hence climbability and sittability should not be considered affordances stricto sensu. Yet we should pay attention to the possibility that the natural language of users includes these verbs as truly functional, by attributing the intention to the object.

For these reasons, the functional affordances lexicon have been built by adding the suffix ability to the stem (the lexical root of the word) of functional verbs using the list of verbs disclosed by Bonaccorsi and Fantoni, (2007). Then it has been expanded using a list of -ility ending terms obtained from WordNet $^{1}$. Finally, through Boolean operation the terms contained within the former group have been filtered out from the latter. At this point an algorithm is applied to the resulting group in order to separate words with verbal roots from words without any verbal root and tagged as properties (similar to the concept of performance (De Benetti et al., 2017)).

The developed lexicon contains 38 functional affordances (e.g. acceptability, adaptability, alterability) and 154 non-functional affordances (e.g. accessibility, accountability, admirability). It was also possible to collect a list of 123 properties ending in -ility (e.g. ability, agility, amability) giving a clear answer to the problem of identifying false positives in the collection of words ending with-ability.

\subsection{Rules to find sentences containing affordances}

The goal of this section is to detect the lexical constructions that are able to identify sentences containing affordances in a given text. This approach helps to detect those affordances that are not

\footnotetext{
${ }^{1}$ Wordnet.com
} 
explicitly expressed neither by the word affordance, or the construct verb+ility, respectively explored in sections 3.1 and 3.2.

After reading a sample of patents, a set of rules is deductively outlined and an algorithm of text mining is implemented to detect the sentences that match these rules. These rules are shown in second column of Table 2, while the first column shows a sample of sentences containing affordances extracted using the relative rules. The sentences where extracted from a random sample of 10,000 patents collected from the freepatent ${ }^{2}$ database. From the entire set, only 398 sentences contained one or more of the rules.

Many of the automatically selected sentences begin with pronouns which relates to the previous part of the patent. In these cases the rules are observed even if the features are not explicitly present in the sentence. For example, the sentence "Due to this, the user can easily recognize the used portion of the tool (4)" is related to the immediately previous sentence in the patent "The used portion (image 122) and the unused portion (image 123) are displayed by mutually different display modes (for example different colors)".

Furthermore, looking at the sentence "The color makes easy to identify the grasping position" suggests the abstraction of some semantic considerations. Indeed, in this sentence the feature (color) that promotes the affordance has an impact on one of the five senses. In addition, there is a construct "easy to" that indicates a design action and there is a verb indicating a function or a learning and cognitive action. All of these abstractions suggest the deployment of a knowledge-based detection system as further future effort.

Table 2. Sentences automatically detected with high likelihood of containing affordance

\begin{tabular}{|l|l|}
\hline Sentence & Rule \\
\hline $\begin{array}{l}\text { The user can easily navigate a set of visual } \\
\text { representations of the earlier views }\end{array}$ & $\begin{array}{l}\text { The term user followed by the modal verb can } \\
\text { and adverbs such as readily, efficiently, quickly } \\
\text { and easily }\end{array}$ \\
\hline $\begin{array}{l}\text { Displaying the individual stats in a similar } \\
\text { manner makes it easier for the user to } \\
\text { read/interpret the group }\end{array}$ & $\begin{array}{l}\text { The verb make followed by the comparative } \\
\text { adjective easier and for user to }\end{array}$ \\
\hline $\begin{array}{l}\text { The map view can include a map filter which } \\
\text { allows the user to quickly sort through providers } \\
\text { on a geographical scale }\end{array}$ & $\begin{array}{l}\text { The verb allow to followed by user and adverbs } \\
\text { such as readily, efficiently, quickly and easily }\end{array}$ \\
\hline $\begin{array}{l}\text { By representing documents as numbers, a user } \\
\text { can easily use a simple keypad to indicate the } \\
\text { document that he wants to print }\end{array}$ & $\begin{array}{l}\text { The term user followed by can and adverbs such } \\
\text { as readily efficiently, quickly and easily }\end{array}$ \\
\hline $\begin{array}{l}\text { Superficial burns are easy to clinically diagnose } \\
\text { by eye and to treat with salves and dressings }\end{array}$ & Forms of the verb to be combined with easy to \\
\hline $\begin{array}{l}\text { A user may proactively identify himself or herself } \\
\text { by entering self-identification information via a } \\
\text { user interface of the user device }\end{array}$ & $\begin{array}{l}\text { The term user followed by the modal verb may } \\
\text { and a verb which indicates the human ability to } \\
\text { detect something }\end{array}$ \\
\hline $\begin{array}{l}\text { The interface enables a user to more easily view } \\
\text { recent notes and emails associated with a } \\
\text { particular client }\end{array}$ & $\begin{array}{l}\text { The verb enable to combined with user and } \\
\text { adverbs such as readily, efficiently, quickly and } \\
\text { easily }\end{array}$ \\
\hline
\end{tabular}

\section{A VALUABLE PRELIMINARY RESULT: AFFORDANCES FOR DISABLED PEOPLE}

If the affordances of an artifact are the set of all potential human behavioral interactions that the artifact itself may allow, disability implies that only a subset of possible interactions are permitted, with respect to the average user.

\footnotetext{
${ }^{2}$ Freepatents.com
} 
It is possible to suppose that human senses functioning is similar to a process, in which each element of a sensory organ performs a biological function. If the sense does not work (e.g. a blind person) or works wrongly (e.g. a color-blind person), the whole or part of the sensory process will be compromised. In some cases correcting the sense process malfunction is impossible or too expensive (at least nowadays). How can affordances help a person with a non-functioning sense?

Human beings normally perceive things throughout the five senses. However, when a sense doesn't work (correclty or at all), the perception continues to be made using remaning senses. Therefore disabled people transfer the perception of things from a non-functioning sense to a functioning one. Sensory sobstitution is acknowledged in patent literature: new additional features are designed in inventions intended to disbaled people. Examples of such additional features (e.g. for visual or hearing impaired people) can be especially found in patents. Considering the sight process malfunctions, it is possible to find tactile and auditive solutions which makes disable people see via ears that can be considered as "eyes".

Within the group of visual impaired people, there are also the color-blind ones, that could be enabled to see colors using particular combinations of different forms and lights. An example can be found in the following patent example (see Figure 2) extracted using the rules shown in section 3.3 : "The bar shape can be formed by either covering portions of a conventional incandescent traffic light or LED traffic light, or the LEDs can be arranged in two or three rows to form the horizontal bar. Because the bar is illuminated red, ordinary drivers are able to distinguish the color and stop. Because the light forms a horizontal bar, color blind drivers are able to easily determine that the light is signaling a stop".

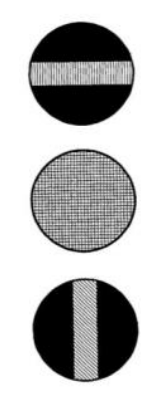

Figure 2. Traffic control system, United States Patent 8154423

In this example it is possible to see a specific affordance for color-blind people. However, considering the hearing malfunctions, it is not possible to reach similar conclusions. Searching in the full text of patents it is not possible to find explicit expressions of affordances for hearing impaired people with respect to sample that we analyzed in the present paper. It seems that the distribution of affordances across the five senses is not uniform, so that some senses prevail over others. Probably if humans had perceived the world throughout hearing like bats, in patents there would be a different distribution of affordances for hearing impaired people.

\section{CONCLUSION AND FUTURE DEVELOPMENTS}

The paper offers a preliminary discussion of a new methodology to extract affordance-related expressions from technical document in automated way. It suggests to use the full text of patents as the main source of information. It develops a set of three approaches that identifies regular linguistic constructs and abstract rules for the identification of affordance-related expressions.

The methodologies significantly improves over the existing literature, which is largely based on case studies and experimental studies, usually involving a small subset of affordances. It is shown that these words include many false positives and suggests that an appropriate subset of words ending with -ability can be defined by restricting to those including functional verbs. It is also shown that limiting the search to words ending with the -ability suffix generates false negatives, or affordance-related expressions that are formed by several words simply because the English language pronunciation does not permit to collapse the meaning into one word.

On the basis of this methodology, an extensive search for affordance-related expressions is carried out in the full text of patents. 
It has also been shown that the number of patents including explicitly the word "affordance" and "user" are correlated, but for new and emerging technologies the frequency of user-related words largely outnumbers the affordance-related frequency. This is a hint to the dynamic process of affordance creation, which requires extensive user experience before becoming an automatic cognitive mechanism.

Finally, the conditions for creating practical tools to support the designer activity are identified. Searching for statistically validated correspondences between features of objects and users' immediate experiences will deliver a large collection of suggestions for practical use by designers.

\section{REFERENCES}

Bonaccorsi, A. and Fantoni, G. (2007), "Expanding the functional ontology in conceptual design”, In: International Conference on Engineering Design. Citeseer.

Bonino, D. Ciaramella, A. and Corno, F. (2010), "Review of the state-of-the-art in patent information and forthcoming evolutions in intelligent patent informatics", World Patent Information, Vol. 32 No. 130-38.

Boyack, K.W., Small, H. and Klavans, R. (2013), "Improving the accuracy of co-citation clustering using full text", Journal of the American Society for Information Science and Technology, Vol. 64 No. 9, pp. 1759-1767.

Brown, D.C. and Blessing, L. (2005), "The relationship between function and affordance", In: ASME 2005 International Design Engineering Technical Conferences and Computers and Information in Engineering Conference, American Society of Mechanical Engineers, pp. 155-160.

Cascini, G., Del Frate, L., Fantoni, G. and Montagna, F. (2011), "Beyond the design perspective of Gero's FBS framework", In: Design computing and cognition, Springer, Vol. 10, pp. 77-96.

Chemero. A. (2003), "An outline of a theory of affordances", Ecological psychology, Vol. 15 No. 2, pp. 181-195.

De Benetti, N., Fantoni, G., Chiarello, F., Bonaccorsi, A., Fadel, G. and Mata, I. (2017), "On the relationship between affordance and expected performance", In: DS 87-4 Proceedings of the 21 st International Conference on Engineering Design (ICED 17) Vol 4: Design Methods and Tools, Vancouver, Canada, 2125.08. 2017.

Dong, H. and Vanns, N. (2009), "Designing an innovative pill dispenser: An undergraduate level case study of inclusive design", The Design Journal, Vol. 12 No. 1, pp. 95-115.

Ernst, H. (2003), "Patent information for strategic technology management", World patent information, Vol. 25 No. 3, pp. 233-242.

Gaver, W.W. (1991), "Technology affordances”, In: Proceedings of the SIGCHI conference on Human factors in computing systems, ACM, pp. 79-84.

Gero J.S. and Kannengiesser, U. (2004), "The situated function-behaviour-structure framework", Design studies, Vol. 25 No. 4, pp. 373-391.

Gibson, J.J. (1996), "The senses considered as perceptual systems".

Gibson, J.J. (2014), “The ecological approach to visual perception: classic edition”, Psychology Press.

Gibson, K.R., Gibson K.R. and Ingold, T. (1994), "Tools, language and cognition in human evolution", Cambridge University Press.

Goodman-Deane, J., Waller, S., Bradley, M., Yoxall, A., Wiggins, D. and Clarkson, P. J. (2016), "Designing Inclusive Packaging", In: Integrating the Packaging and Product Experience in Food and Beverages, Woodhead Publishing, pp. 37-57.

Jamone, L. Ugur, E., Cangelosi, A., Fadiga, L., Bernardino, A., Piater, J. and Santos-Victor, J. (2018), "Affordances in psychology, neuroscience, and robotics: A survey", IEEE Transactions on Cognitive and Developmental Systems, Vol. 10, No. 1, pp. 4-25,.

Kannengiesser, U. and Gero, J.S (2012), “A process framework of affordances in design”, Design Issues, Vol. 28 No. 1 , pp. 50-62.

Langdon, P., Johnson, D., Huppert, F. and Clarkson, P. J. (2015), “A framework for collecting inclusive design data for the UK population", Applied ergonomics, Vol. 46, pp. 318-324.

Maier, J.R.A. and Fadel, G.M. (2003), “Affordance-based methods for design”, In: ASME 2003 International Design Engineering Technical Conferences and Computers and Information in Engineering Conference, American Society of Mechanical Engineers, pp. 785-794.

Masahiro, M. (1970), "Bukimi no tani - The uncanny valley", Energy, (MacDorman, K. F. and Minato, T., Trans.), Vol. 7 No. 4, pp. 33-35.

Nagel, J.K.S., Stone, R.B., and McAdams, D.A. (2010), "An engineering-to-biology thesaurus for engineering design", In: ASME 2010 international design engineering technical conferences and computers and information in engineering conference, American Society of Mechanical Engineers, pp. 117-128.

Norman. D. A. (1999), “Affordance, conventions, and design”, interactions, Vol. 6 No. 3, pp. 38-43. 
Pahl G. and Beitz, W. (2013), "Engineering design: a systematic approach”, Springer Science and Business Media.

Savransky, S.D. (2000), "Engineering of creativity: Introduction to TRIZ methodology of inventive problem solving". CRC Press.

Stoffregen, T.A. (2003), “Affordances as properties of the animal-environment system", Ecological psychology, Vol. 15 No. 2 pp. 115-134.

Spreafico, C., Fantoni, G. and Russo, D. (2015), "Fbs models: an attempt at reconciliation towards a common representation", In: proceedings of the International Conference on Engineering Design, Vol. 2, Design Society, p. 399-408.

Stone, R.B. and Wood, K. L. (2000), "Development of a functional basis for design", Journal of Mechanical design, Vol. 122 No. 4, pp. 359-370.

Turvey, M.T. (1992), “Affordances and prospective control: An outline of the ontology”, Ecological psychology, Vol. 4 No. 3, pp. 173-187.

Yuen-Hsien, T., Chi-Jen, L. and Yu-I, L. (2007), "Text mining techniques for patent analysis", Information Processing \& Management, Vol. 43 No. 5, pp. 1216-1247. 